\title{
Development of a very small telescope for a milli-arcsec space astrometry
}

\author{
M. Suganuma ${ }^{1} \dagger$, Y. Kobayashi ${ }^{1}$, N. Gouda ${ }^{1}$, \\ T. Yano ${ }^{1}$, Y. Yamada ${ }^{2}$, N. Takato ${ }^{3}$ \\ and M. Yamauchi ${ }^{4}$ \\ ${ }^{1}$ National Astronomical Observatory of Japan, 2-21-1 Osawa, Mitaka, Tokyo 181-8588, Japan \\ email: suganuma@merope.mtk.nao.ac.jp \\ ${ }^{2}$ Department of Physics, Kyoto University, Oiwake-cho Kita-shirakawa, Sakyo-ku, Kyoto \\ 606-8502, Japan \\ ${ }^{3}$ Subaru Telescope, National Astronomical Observatory of Japan, 650 North A'ohoku Place, \\ Hilo, HI 96720, USA \\ ${ }^{4}$ Department of Astronomy, Graduate School of Science, The University of Tokyo, 7-3-1 \\ Hongo, Bunkyo-ku, Tokyo 113-0033, Japan
}

\begin{abstract}
We are preparing a reflecting telescope for Nano-JASMINE, a very small satellite for global space astrometry of milli-arcsecond accuracy. The telescope has a 5-cm diameter primary mirror and a beam-combiner in front of it. It occupies only about $12 \times 12 \times 17 \mathrm{~cm}$ and is entirely made out of aluminum alloy. The telescope and its surrounding structures are carefully designed for thermal stability of the optics, especially to control changes in the relative angle of the beam-combiner.
\end{abstract}

Keywords. astrometry, space vehicles: instruments, telescopes

\section{The Nano-JASMINE Telescope}

Nano-JASMINE satellite, which weighs about $14 \mathrm{~kg}$, will survey all-sky in wavelength around $z$-band using a CCD in time-delayed-integration (TDI) mode. In this mission, we are going to demonstrate a global astrometry observation with a small satellite. In the case of success, a new astrometric catalog with a few mas accuracies could produce proper motions accurate to 0.1 mas $\mathrm{yr}^{-1}$, when combining with Hipparcos catalog (Kobayashi et al. 2006).

For Nano-JASMINE, we are developing a very small telescope that is specified in Table 1. Similar to Hipparcos, we place a dual-angled flat mirror, called beam-combiner, in front of the primary mirror so that we could simultaneously expose two different field-of-views separated by 99.5 degrees in order to execute wide-field astrometry.

\section{Developement and current status}

We made all telescope parts, including the mirrors, out of aluminum alloy using an ultra-precise milling machine, however, we could not fabricate aspherical mirrors satisfactory (for details, see Suganuma et al. 2006). Figure 1 shows a picture of a proto-model of the telescope, together with its optical layout. A diffraction-limited performance of the telescope optics was confirmed both by wavefront measurements and imaging experiments.

$\dagger$ Present address: National Astronomical Observatory of Japan, 2-21-1 Osawa, Mitaka, Tokyo 181-8588, Japan. 
Table 1. Specifications of Nano-JASMINE Telescope

\begin{tabular}{l|l}
\hline Effective Aperture & $\phi=5 \mathrm{~cm}$, divided into two by a beam-combiner. \\
Focal Length & $167 \mathrm{~cm}(\mathrm{~F} / 33)$ \\
Optics Type & Ritchey-Chretien type, followed by three folding mirrors. \\
Field of View & $0.5 \times 0.5 \mathrm{deg}$ \\
Basic Angle & $99.5 \mathrm{deg}$ \\
Wavelength & $z$-band $(\lambda \sim 0.9 \mu)$ \\
CCD & $1024 \times 1024 \mathrm{pix}(1.76 \mathrm{arcsec} / \mathrm{pix})$ \\
Operating Temperature & $-50--100^{\circ} \mathrm{C}$ \\
\hline
\end{tabular}
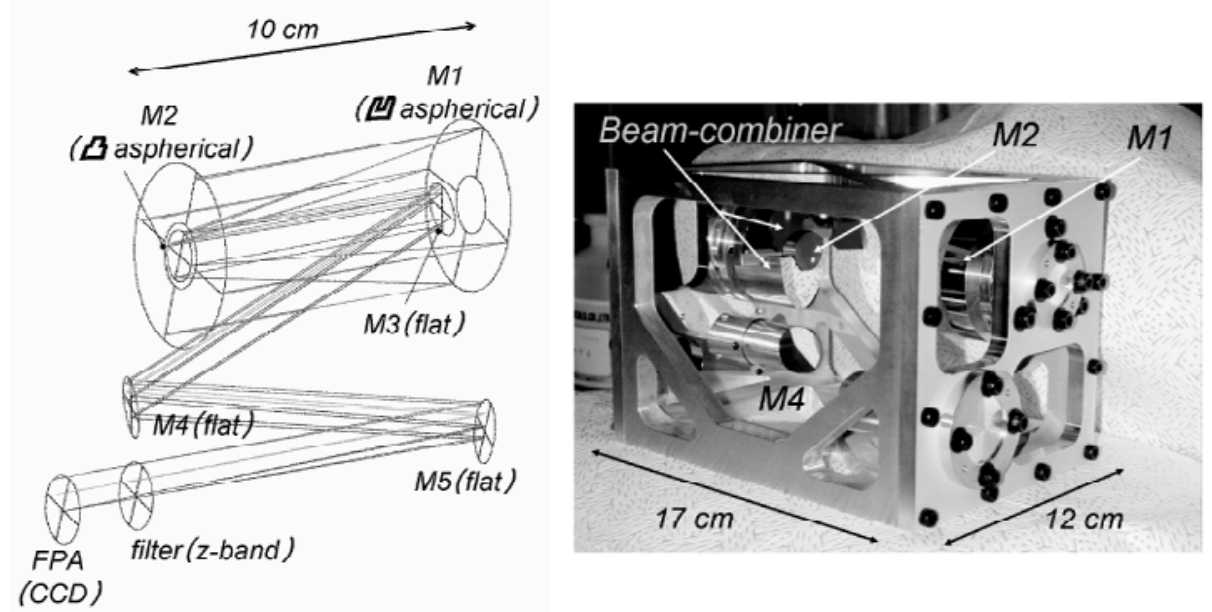

Figure 1. Left: Optical layout of Nano-JASMINE telescope. A beam-combiner that should appear around the M2 is omitted here. Right: Assembled proto-model of the telescope. All parts are figured out of aluminum alloy except the gold coat on the optical reflecting surfaces.

The surrounding structures are designed to cool down the telescope and the CCD radiatively below $-50^{\circ} \mathrm{C}$. Also, the telescope is well-insulated from exterior structures to minimize the thermal gradients in the optics and their time variation during the orbital period of the satellite. We made a realistic thermal design that passively controls the changes in the relative angle of the beam-combiner within a sub-milliarcsecond.

\section{Acknowledgements}

The Nano-JASMINE project is collaborated with Intelligent Space Systems Laboratory, Univ. of Tokyo. Our development is supported by the Advanced Technology Center at the National Astronomical Observatory of Japan. The practical machining process was carried out by Corporate Manufacturing Engineering Center of TOSHIBA Co., Ltd.. We thank IHI Aerospace Engineering Co., LTD. for their thermal analysis and constructive suggestions on the thermal design. We also acknowledge colleagues of JASMINE working group for helpful discussions.

\section{References}

Kobayashi, Y. et al. 2006, Proc. SPIE 6265, 626544

Suganuma, M., Kobayashi, Y., Gouda, N., Yano, T., Yamada, Y., Takato, N., \& Yamauchi, M. 2006, Proc. SPIE 6265, 626545 